\title{
Dense Metal Plasma in a Solenoid for Ion Beam Neutralization
}

\author{
André Anders, ${ }^{1}$ Fellow, Marina Kauffeldt, ${ }^{2}$ Efim M. Oks, ${ }^{3}$ and Prabir K. Roy, ${ }^{1}$ Member \\ ${ }^{1}$ Lawrence Berkeley National Laboratory, 1 Cyclotron Road, Berkeley, California 94720, USA \\ ${ }^{2}$ University of the German Federal Armed Forces, Werner-Heisenberg-Weg 39, 85577 \\ Neubiberg, Germany \\ ${ }^{3}$ High Current Electronics Institute, 2/3 Academichesky Ave, Tomsk, 634055, Russia
}

\section{ACKNOWLEDGMENT}

Support by E. Gilson, P. Seidl, W. Greenway, M. Dickinson, S. Lidia, J.-Y. Jung, B. G. Logan, J. Wallig, and J. Schein is gratefully acknowledged. M.K. thanks for financial support by the Deutscher Akademischer Austauschdienst (DAAD), E.M.O. was supported by project T2-196 of the Initiatives for Proliferation Prevention of the U.S. Department of Energy. Work at Berkeley Lab was supported by the U.S. Department of Energy, and under Contract No. DE-AC02-05CH11231.

\section{DISCLAIMER}

This document was prepared as an account of work sponsored by the United States Government. While this document is believed to contain correct information, neither the United States Government nor any agency thereof, nor The Regents of the University of California, nor any of their employees, makes any warranty, express or implied, or assumes any legal responsibility for the accuracy, completeness, or usefulness of any information, apparatus, product, or process disclosed, or represents that its use would not infringe privately owned rights. Reference herein to any specific commercial product, process, or service by its trade name, trademark, manufacturer, or otherwise, does not necessarily constitute or imply its endorsement, recommendation, or favoring by the United States Government or any agency thereof, or The Regents of the University of California. The views and opinions of authors expressed herein do not necessarily state or reflect those of the United States Government or any agency thereof or The Regents of the University of California. 


\title{
Dense Metal Plasma in a Solenoid for Ion Beam Neutralization
}

\author{
André Anders, ${ }^{1}$ Fellow, Marina Kauffeldt, ${ }^{2}$ Efim M. Oks, ${ }^{3}$ and Prabir K. Roy, ${ }^{1}$ Member \\ ${ }^{1}$ Lawrence Berkeley National Laboratory, 1 Cyclotron Road, Berkeley, California 94720, USA \\ ${ }^{2}$ University of the German Federal Armed Forces, Werner-Heisenberg-Weg 39, 85577 \\ Neubiberg, Germany \\ ${ }^{3}$ High Current Electronics Institute, 2/3 Academichesky Ave, Tomsk, 634055, Russia
}

\begin{abstract}
Space-charge neutralization is required to compress and focus a pulsed, high-current ion beam on a target for warm dense matter physics or heavy ion fusion experiments. We described approaches to produce dense plasma in and near the final focusing solenoid through which the ion beam travels, thereby providing an opportunity for the beam to acquire the necessary space-charge compensating electrons. Among the options are plasma injection from pulsed vacuum arc sources located outside the solenoid, and using a high current ( $>4 \mathrm{kA}$ ) pulsed vacuum arc plasma from a ring cathode near the edge of the solenoid. The plasma distribution is characterized by photographic means, by an array of movable Langmuir probes, by a small single probe, and by evaluating Stark broadening of the Balmer $\mathrm{H}_{\beta}$ spectral line. In the main approach described here, the plasma is produced at several cathode spots distributed azimuthally on the ring cathode. It is shown that the plasma is essentially hollow, as determined by the structure of the magnetic field, though the plasma density exceeds $10^{14} \mathrm{~cm}^{-3}$ in practically all zones of the solenoid volume if the ring electrode is placed a few centimeters off the center of the solenoid. The plasma is non-uniform and fluctuating, however, since its density exceeds the ion beam density it is believed that this approach could provide a practical solution to the space charge neutralization challenge.
\end{abstract}




\section{INTRODUCTION}

For warm dense matter and ion-beam-driven inertial fusion experiments [1-2], high current pulsed ion beams are used to heat and compress a target of interest [3-5]. While many beams will be needed to achieve inertial fusion, current experiments aim at the development of a single beam of heavy ions such as $\mathrm{K}^{+}$or $\mathrm{Li}^{+}$(here, "heavy" is understood in comparison to the mass of a proton). The ion beam needs to be compressed radially and longitudinally to reach on target a current in the tens of ampere range and with a pulse duration of 1 ns [6-7]. A very strong space charge would build up as the beam is compressed unless a means of ion space charge compensation is provided.

Space charge compensation can be achieved when the ion beam travels through plasma whose density exceeds the beam density [8]. Therefore, linear heavy ion accelerators designs incorporate one or several plasma generator(s) placed near and inside the finial focusing solenoid (FFS). In previous efforts, this included a ferroelectric plasma source [9] upstream of the FFS, and four cathodic arc plasma sources (arc plasma "miniguns" [10]) which injected plasma from downstream of the FFS [5]. The use of cathodic vacuum arc plasma is appealing [11] because cathode spots are known to be prolific generators of dense metal plasma that can pump itself by condensing on the chamber walls [12-13]. Hence the formation of dense plasma does not lead to any significant gas load in the accelerator system.

The original idea of filling the solenoid was using the fast flowing vacuum arc plasma and injecting it along the magnetic field lines into the solenoid (Fig. 1). One would make use of the fact that plasma tends to stream along field lines. This streaming is facilitated by the "magnetization" of electrons: the Lorentz force makes them gyrate around the magnetic field lines, which does not affect mobility along field lines but greatly limits mobility across field lines. The ions of the plasma are also bound to the field lines. In the case of strong fields, their gyration radius $r_{g, i}=m_{i} u_{i \perp} / Q e B$ is small compared to a characteristic length, like the inner solenoid radius, and they can be considered magnetized like the electrons $\left(m_{i}\right.$ is the ion mass, $u_{i \perp}$ the ion velocity component perpendicular to the magnetic field vector, $Q$ is the charge state number, which is most often 2 or even 3 for vacuum arc plasmas, $e$ is the elementary charge, and $B$ is the magnetic induction). However, even if ions are not magnetized, like in the weaker magnetic field zone outside the solenoid, their motion is still bound to the field lines due to the electric field that arises from the electron-ion interactions [13-14].

For symmetry reasons, two [11] or four [5] miniature plasma sources were used, which had previously been developed for pulsed cathodic arc deposition of thin films [10, 13]. The number four was a compromise between having as many sources as possible distributed azimuthally, and practical limits dictated by space constrains and cost. Fig. 2 shows the four 
arc sources on a common mounting flange, and Fig. 3 shows the plasma as seen through a vacuum window.

It is clear that a number of issues have to be resolved towards the goals of filling the whole volume of the solenoid with dense plasma. Among them are the magnetic mirror effect [15] which limits the amount of plasma that can enter a region of high magnetic field strength. Additionally, we notice that there is a limited availability of magnetic field lines that connect the arc sources with the volume defined by the inner diameter of the solenoid without intersecting the vacuum chamber wall and other components (Fig 1). Following those field lines not intersecting any hardware one can see that only the central region of the solenoid volume is reached, and hence only the central axial region should receive dense plasma. All other plasma is guided to the wall and will condense there. Indeed, one can find a coating on the wall as shown in Fig. 4 (as a side effect, this figure gives an indication that the azimuthal distribution is quite uniform despite using only four sources). The plasma distribution inside the solenoid was investigated using a movable array of 37 Langmuir probes operating in ion saturation mode, confirming the expectations that the plasma density is concentrated near the axis of the solenoid while a significant zone between axis and inner wall of the solenoid is void of dense plasma [16].

Results obtained at the Neutralized Drift Compression Experiment (NDCX) at Berkeley Lab showed 60-fold longitudinal compression of a $300 \mathrm{keV} \mathrm{K}^{+}$beam when using plasma-based space charge neutralization [5, 11]. The next generation drift compression experiment, NDCX-II [7], currently under construction at Berkeley Lab, requires improved neutralization for higher beam currents, which implies higher plasma densities $\left(>10^{14} \mathrm{~cm}^{-3}\right)$ and a more complete filling of the solenoid volume and the region between the FFS and the beam's target. To achieve this goal, we consider in this contribution a different approach involving a ring cathode vacuum arc source placed inside the FFS. The resulting plasma distribution will be characterized by photographic, probe, and spectroscopic techniques.

\section{DISCHARGE SYSTEM}

In contrast to the previous experiments [16], where plasma was made at four arc "mini guns" external to the solenoid, arc plasma is generated here on a ring cathode place inside the solenoid as indicated in Figs. 5 and 6. The grounded vacuum pipe served as the anode of the discharge, and the cathode ring was separated from the pipe by a ceramic (alumina) tube. The inner diameter of the cathode ring was $25 \mathrm{~mm}$, which is just large enough to accommodate the ion beam as it enters the FFS region.

Cathodic arc plasma is generated at very small, non-stationary cathode spots [13]. The ignition and extinction of spots make the spot appear to move on the cathode surface. Initial arc ignition is facilitated by a slightly conductive layer on the front face of the insulator separating 
cathode and anode. Due to the absence of a dedicated trigger electrode, this ignition approach is sometimes called "triggerless" ignition [17]. The plasma density is highly non-uniform: it is extremely dense near the spot $\left(\sim 10^{20} \mathrm{~cm}^{-3}\right.$ have been observed [18-19]), and it expands with $10^{4} \mathrm{~m} / \mathrm{s}$ into the vacuum [20].

The magnetic field was generated by the solenoid designed to be pulsed with high current up to $8.4 \mathrm{kA}$, delivering a magnetic induction in the center of the coil up to 3 Tesla in these experiments, and up to 8 Tesla when NDCX-II is fully operational. The solenoid current had a half-sine shape with a duration of $1 \mathrm{~ms}$ FWHM (full width at half maximum) supplied by a thyristor-switched $8 \mathrm{mF}$ capacitor. The maximum charging voltage applied to the capacitor was $700 \mathrm{~V}$.

The arc current pulse was much shorter than the solenoid pulse, about $100 \mu \mathrm{s}$, fed by a thyristor-switched pulse-forming network (PFN). The flat portion of the pulse had a maximum of $4.5 \mathrm{kA}$ when the PFN was charged to the maximum of $1 \mathrm{kV}$. The arc discharge was delayed relative to the solenoid pulse such as to operate at maximum magnetic field. The magnetic field can be considered to be approximately constant during the arc pulse. The ion beam (not actually used in these experiments) has a duration of several nanoseconds, and therefore both arc plasma and magnetic field can be considered constant on that timescale. The pulse repetition rate was varied but was generally low, about 1 pulse per 10 seconds, limited by the recharging time of the capacitors and cooling concerns of the FFS coil. The typical wave forms of the coil current and arc current are shown in Fig. 7, with the arc voltage (as measured at the cathode feedthrough) shown, too. We see that the peak power making the plasma (product of arc current and voltage) is about $17.5 \mathrm{~kW}$.

The cathode material could be selected freely and may represent one component that can be optimized in later studies. For this preliminary study we used copper, stainless steel, and aluminum.

The cathode ring was slit in one place along the axial direction in order to prevent an azimuthal current that would be induced when pulsing the coil. Without the slit the cathode ring would represent a single-turn secondary of a transformer where the solenoid coil is the

primary. Azimuthal current in the ring is highly undesirable because it would weaken the beam-focusing magnetic field.

\section{PLASMA DIAGNOSTICS}

\section{A. Photography}

The plasma was first studied using photography in order to obtain qualitative information on the plasma distribution. For this purpose, a mirror was inserted such as to reflect emitted light through a vacuum window as indicated in Fig. 5. Besides making convenient use of the 
window, the mirror is also used to address a possible issue of window coating by the condensing arc metal plasma. Metal condensation and coating on the mirror (as opposed to condensing on the window) has practically no consequences for maintaining visual access to the discharge. The images were taken with a digital Canon EOS 300D single lens reflex camera, and an attached $200 \mathrm{~mm}$ zoom lens. The camera was operated in open shutter mode to capture light integrated over one full discharge pulse.

\section{B. Movable probe array}

The plasma density inside the solenoid was measured using an array of 37 Langmuir probes, which were already described in [16]. Briefly, thirty-seven 24-gauge solid copper wires were placed in alumina tubes of $1.64 \mathrm{~mm}$ inner diameter and 2.46 outer diameter; they were arranged in a honeycomb pattern, as shown in Fig. 8, with a spacing between the centers of the wires of $2.46 \mathrm{~mm}$. The assembly could be moved axially via a precision translational stage.

Each probe was connected to its circuit via a vacuum feedthrough. A representative of the 37 identical circuits is shown in Fig. 9. Essentially, a capacitor is used to stabilize the applied probe voltage $(-70 \mathrm{~V})$ during the pulse. The current could be picked up using a resistor of suitable resistance, however we opted to utilize a pair of resistors (voltage divider) to limit the current in the case an arcing event occurs on the probe. If the latter happens, the probe sheath resistance practically disappears, the probe current rises sharply, and the charge of the capacitor is drained until it is discharged and the voltage much reduced.

Arcing on the probe is highly undesirable for several reasons. First, the measurement is useless and must be discarded. Second, the plasma made on the probe could damage the probe if it occurs frequently: the probe wire would be eroded and the insulator between probes coated with a metal layer. Third, a large transient current would cause a large transient voltage at the measuring resistor, which could damage the entrance amplifier of the 40-channel oscilloscope. As a precaution we used a voltage divider and additional protective circuitry.

The data recording was done with a National Instruments PXI-1045 Chassis equipped with five PXI-5105 8-channel digitizer cards, with a maximum sampling rate of $60 \mathrm{MS} / \mathrm{second}$ per channel and 12 bit resolution, operated under NI LabVIEW virtual instrument (VI) software. The VI allowed us to set record length, sampling rate, voltage range, and specify the trigger mode. The on-board stored data were transferred to a personal computer for further analysis using a MATLAB script.

The software was written to create equi-density contour plots and animated sequences of the plasma density evolution in one plane, or the time-averaged plasma density in selected planes of the $(\mathrm{x}, \mathrm{y}, \mathrm{z})$ space, where $\mathrm{z}$ is assigned to the beam axis and $(\mathrm{x}, \mathrm{y})$ are planes perpendicular to $\mathrm{z}$. When time-averaging was applied for a given fixed axial z-position of the probes, the averaging 
was done over the period between the half rise and half fall value of the cathodic arc current pulse.

\section{Movable single probe}

The relatively massive 37-probe array can cause significant disturbance of the discharge if the array is placed inside the solenoid. Therefore, in some of the experiments, a single Langmuir probe of overall much smaller dimensions was used that affected the plasma to a much lesser degree. This probe had a special design to maintain a well-defined collection area even as the probe entered a zone of dense metal plasma where coating of the probe oocurs: a small entrance aperture was used, as indicated in Fig. 10. This figure also indicates that the translational mechanism of the 37-probe array was used to position the single probe axially (radial change of the probe position was not possible). The probe bias voltage was again -70 $\mathrm{V}$ to ensure ion saturation, and the data collection and evaluation were analogous to the measurements done with the probe array.

\section{Spectroscopy}

In order to avoid any disturbance of the plasma, density measurements were also done by measuring the Stark broadening of the $\mathrm{H}_{\beta}$ line [21-22] with the probes absent. The idea is to make use of the water vapor that is adsorbed on the cathode and ionized when the arc spot erodes the material from the cathode surface. Unless operation is done in ultrahigh vacuum (UHV), all surfaces are covered with water, hydrocarbons, and other contaminants, which deliver hydrogen to the plasma. The $\mathrm{H}_{\beta}$ line is a much-used spectral line for which line broadening is well investigated [21-23]. Assuming for a moment a uniform plasma and the dominance of Stark broadening over any other broadening mechanism for the $\mathrm{H}_{\beta}$ line, the electron density $n_{e}$ (in $\mathrm{cm}^{-3}$ ) can be determined by

$$
\Delta \lambda_{1 / 2}=2.5 \times 10^{-10} \alpha_{1 / 2} n_{e}^{2 / 3}
$$

simply by measuring the full width at half maximum (FWHM) $\Delta \lambda_{1 / 2}$ (in $\mathrm{nm}$ ); the shape

parameter $\alpha_{1 / 2}$ is $0.08 \pm 0.02$ for the $\mathrm{H}_{\beta}$ line [22, 24].

The spectrum emitted from the plasma in the solenoid was collected using two spectrometers, first by a Telemark PMS 420 (spectral range $350 \mathrm{~nm}$ to $1050 \mathrm{~nm}$, $\Delta \lambda \sim 0.05 \mathrm{~nm}$ at $\lambda=500 \mathrm{~nm}$ ) for the overview spectrum, and by a Jobin Yvon TH 1500 for the high resolution measurements $(\Delta \lambda \sim 0.0015 \mathrm{~nm}$ at $\lambda=500 \mathrm{~nm})$. A similar setup as for photography was used, except that the plasma region of interest was now imaged onto the 
entrance fiber (PMS 420) or entrance slit (TH 1500), respectively. The optical path could be changed via a movable mirror outside the vacuum such that light from a hollow-cathode spectral lamp (mercury, hydrogen) could be directed to the entrance fiber/slit of the spectrometer in use, and thus a wavelength calibration could be performed as it is common in emission spectroscopy.

With the help of the calibration, the number of detector pixels per nanometer of wavelength could be determined (namely $0.0061 \mathrm{~nm} /$ pixel for in the region near $\lambda=500 \mathrm{~nm}$ for the TH1500's retrofitted CMOS-detector), which is necessary when evaluating the line broadening from

plasmas. It is known that the line widths of hollow cathode spectral lamps are very narrow and that measurements with those lamps indicate instrumental line broadening. The measurements with the spectral lamps are therefore used to make sure that instrumental line broadening is much smaller than the broadening of the $\mathrm{H}_{\beta}$ line as emitted from the arc plasma. Given the error associated with plasma non-uniformity along the optical line-of-sight, it was decided that a deconvolution of the measured lines with the instrumental line width is not necessary since it would not really increase the accuracy of the plasma density values obtained.

The cathode material in those experiments were stainless steel (SS) and aluminum, both of which clearly showed the Balmer $\mathrm{H}_{\alpha}(\lambda=656.281 \mathrm{~nm})$ and $\mathrm{H}_{\beta}(\lambda=486.134 \mathrm{~nm})$ lines besides the lines of neutral and ionized metals of the cathode. For the high resolution spectrometer, time-integration over typically five pulses was necessary to obtain an acceptable signal to noise ratio at the detector.

\section{RESULTS}

\section{A. Photography}

Although the emitted light is not strictly proportional to the plasma density due to spatially different excitation conditions, the distribution of the emitted light can provide valuable information on the plasma distribution.

First, photography clearly shows the cathode spots as the sources of plasma. At relatively low current of less than $1 \mathrm{kA}$, the number of spots is limited and the plasma does not fill the volume azimuthally (Fig. 11). An increase of the discharge current to $4.5 \mathrm{kA}$ increases the number of spots such that the plasma appears azimuthally distributed (Fig. 12). One can see well-defined, relatively intense and large spots, commonly labeled as type 2 spots, and multitudes of less intense, not resolved (type 1) spots. An image taken at intermediate current (Fig. 13) nicely illustrates the path of the plasma after it is produced at a cathode (type 2) spot: as expected, the plasma essentially follows the curved magnetic field lines intersecting the emitting spot. Following the field line, the plasma electrons reach the vacuum pipe, which serves as the anode of the discharge. This already makes clear that one can expect a great disturbance of the discharge if the curved path is blocked by inserting the relatively massive structure of the 
37-probe array. The images suggest that measurements with the 37-probe array should be limited to the region outside the paths defined by curved field lines from cathode spots to the pipe wall.

\section{B. Movable probe array}

Since the flowing plasma is closely bound to the magnetic field lines, it is important to place the source of plasma, the cathode spots, at a suitable location such as to guide the plasma to regions where needed. In general, the further the ring of cathode spots is placed away from the center of the solenoid the closer the plasma can arch toward the center axis. Figure 14 shows one example of measurements with the cathode edge $6.5 \mathrm{~cm}$ from the solenoid center, and the probe array positioned $3.5 \mathrm{~cm}$ from the center in the opposite direction (i.e. plasma is streaming towards the probe array). One can clearly see the hollow structure of the plasma distribution.

The "hole" seen in figure 14 can be reduced by further moving the ring cathode away from the center of the solenoid. The peak electron density readily exceeds the desired $10^{14} \mathrm{~cm}^{-3}$, which is greater than the ion beam density. In fact, the peak electron density that has been reached was between 2 and $4 \times 10^{14} \mathrm{~cm}^{-3}$, far exceeding any other previous approach to filling the solenoid volume with plasma.

A number of large data files have been collected, all more or less confirming the previous statement; the data files (movie files) are available from the authors upon request.

As the probe array was further inserted into the solenoid it became apparent that the probe is intersecting the magnetic field lines that connect cathode spots and the anode (vacuum pipe). The discharge may even not operate when the probe is fully blocking the path. No useful measurements could be made this way, which made us move on to single probe measurements whose main purpose was to back-up or disprove results derived from the center probes of the probe array.

\section{Movable single probe}

Not unexpectedly, the results of the small movable single probe generally confirmed the multi-probe measurements. An example is shown in Fig. 15. The important point here is that the targeted density of $10^{14} \mathrm{~cm}^{-3}$ was exceeded in most of the solenoid volume - a major goal of this experiment toward use for beam neutralization. The plasma density is always noisy and we noticed that the noise level increases with increasing magnetic field strength.

\section{Spectroscopy}

The intensity of the hydrogen lines was surprisingly strong, as the overview spectrum of Fig. 16 shows. Identification of the lines was simple using the hydrogen hollow-cathode 
spectral lamp. A higher resolution spectrum, like the one in Fig. 17, shows clearly the $\mathrm{H}_{\beta}$ line at $\lambda=486.1 \mathrm{~nm}$. It is much broader than the other lines of the spectrum, which is another indication that the $\mathrm{H}_{\beta}$ is broadened much beyond the instrumental width of the spectrometer. The line shape indicates a flat top, which is typical for the $\mathrm{H}_{\beta}$ line due the splitting of degenerated sublevels. Self-absorption may also play a role but cannot be very significant given the overall shape [22].

\section{DISCUSSION AND CONCLUSIONS}

Filling of the volume of a solenoid with plasma turned out to be a challenge. The strong effects of the magnetic field on the plasma density distribution and plasma motion leads to peculiar filling effects. For plasma injection from outside the solenoid, the plasma tends to be concentrated on axis in a sharply defined plasma "filament" since off-axis magnetic field lines intersect the vacuum wall and thus do not allow transport to occur. In contrast, plasma produced near the wall inside the solenoid results in a hollow distribution since reaching the axis would imply to cross magnetic field lines. The here-described experiments of producing such plasma are relevant to the task of ion beam neutralization since it was demonstrated that most of the volume can be filled with dense plasma $\left(>10^{14} \mathrm{~cm}^{-3}\right)$ when the source of plasma, the arc spots distributed on a cathode ring, are positioned off-center. Then the plasma follows the arched magnetic field lines and approaches the region near the axis.

The plasma was produced by cathodic arc spots which are prolific generators of plasma by eroding the cathode. Since we do not operate in ultra-high vacuum the erosion involves the cathode material as well as the oxides and adsorbate layers that are likely to be present on the cathode surface. The resulting plasma contains metal and gas species, including hydrogen, which was used for the spectroscopic measurements using Stark broadening of the $\mathrm{H}_{\beta}$ line. Spectroscopic measurements were the least intrusive measurements, while probe measurements always disturb the plasma, especially when a large array was placed in the electron path from cathode to anode.

All of the measurements indicated that the plasma density is greater than $10^{14} \mathrm{~cm}^{-3}$ at least for some time during the arc pulse. This is sufficient for beam neutralization as long as the timing of the beam is optimized such that the beam travels through the solenoid when the plasma density is highest. A quantitative analysis of the broadening mechanisms revealed that Stark broadening is indeed dominant although Doppler broadening should not be completely ignored: about $15-20 \%$ of the broadening can be assigned to the latter. This, however, does not change the main conclusion of this contribution, namely that plasma has been produced in the solenoid with the plasma density exceeding the required minimum value of $10^{14} \mathrm{~cm}^{-3}$.

The previous approach to filling the solenoid with plasma using four cathodic plasma 
sources (Figs. 2 and 3) external to the solenoid-enclosed volume delivered a plasma concentrated on axis. The here-described approach using a ring cathode inside the solenoid resulted in a plasma that is essentially hollow. This suggests that in the future one may use a combination of the two. The approximate optimum position of the ring cathode appears to be about $8 \mathrm{~cm}$ from the center for our configuration. The remaining non-uniformity of the filling may be acceptable because we can guarantee that when the nanosecond ion beam passes through the solenoid, the plasma electron density is greater than the ion beam density at all positions inside the solenoid, and thus space charge compensation can occur. With this argument, we can also accept that the arc plasma is generally "noisy", i.e. fluctuates in time and space due to the explosive, fractal nature of plasma production at cathode spots [13].

Beam neutralization experiments have not yet been made with this new plasma generation concept but are considered among other options.

\section{ACKNOWLEDGMENTS}

Support by E. Gilson, P. Seidl, W. Greenway, M. Dickinson, S. Lidia, J.-Y. Jung, B. G. Logan, J. Wallig, and J. Schein is gratefully acknowledged. M.K. thanks for financial support by the Deutscher Akademischer Austauschdienst (DAAD), E.M.O. was supported by project T2-196 of the Initiatives for Proliferation Prevention of the U.S. Department of Energy. Work at Berkeley Lab was supported by the U.S. Department of Energy, and under Contract No. DE-AC02-05CH11231. 


\section{REFERENCES}

[1] D. R. Welch, D. V. Rose, B. V. Oliver, and R. E. Clark, "Simulation techniques for heavy ion fusion chamber transport," Nucl. Instr. Meth. Phys. Res. A, vol. 464, pp. 134-139, 2001.

[2] J. J. Barnard, J. Armijo, D. S. Bailey, A. Friedman, F. M. Bieniosek, E. Henestroza, I. Kaganovich, P. T. Leung, B. G. Logan, M. M. Marinak, R. M. More, S. F. Ng, G. E. Penn, L. J. Perkins, S. Veitzer, J. S. Wurtele, S. S. Yu, and A. B. Zylstra, "Ion beam heated target simulations for warm dense matter physics and inertial fusion energy," Nucl. Instr. Meth. Phys. Res. A, vol. 606, pp. 134-138, 2009.

[3] A. Kozyreva, M. Basko, F. B. Rosmej, T. Schlegel, A. Tauschwitz, and D. H. H. Hoffmann, "Dynamic confinement of targets heated quasi-isochorically with heavy ion beams," Phys. Rev. E, vol. 68, p. 056406, 2003.

[4] B. G. Logan, L. J. Perkins, and J. J. Barnard, "Direct drive heavy-ion-beam inertial fusion at high coupling efficiency," Phys. Plasmas, vol. 15, pp. 072701-7, 2008.

[5] A. B. Sefkow, R. C. Davidson, E. P. Gilson, I. D. Kaganovich, A. Anders, J. E. Coleman, M. Leitner, S. M. Lidia, P. K. Roy, P. A. Seidl, W. L. Waldron, S. S. Yu, and D. R. Welch, "Simulations and experiments of intense ion beam current density compression in space and time," Phys. Plasmas, vol. 16, pp. 056701-11, 2009.

[6] P. A. Seidl, A. Anders, F. M. Bieniosek, J. J. Barnard, J. Calanog, A. X. Chen, R. H. Cohen, J. E. Coleman, M. Dorf, E. P. Gilson, D. P. Grote, J. Y. Jung, M. Leitner, S. M. Lidia, B. G. Logan, P. Ni, P. K. Roy, K. Van den Bogert, W. L. Waldron, and D. R. Welch, "Progress in beam focusing and compression for warm-dense matter experiments," Nucl. Instrum. Meth. Phys. Res. A, vol. 606, pp. 75-82, 2009.

[7] J. J. Barnard, J. Armijo, F. M. Bieniosek, A. Friedman, M. Hay, E. Henestroza, B. G. Logan, R. M. More, P. A. Ni, L. J. Perkins, S.-F. Ng, J. S. Wurtele, S. S. Yu, and A. B. Zylstra, "Simulations for experimental study of warm dense matter and inertial fusion energy applications on NDCX-II," J. Phys. Conf. Ser., vol. 244, p. 032027, 2010.

[8] B. G. Logan and D. A. Callahan, "Beam charge and current neutralization of high-charge-state heavy ions," Nucl. Instr. Meth. Phys. Res. A, vol. 415, pp. 468-472, 1998.

[9] P. C. Efthimion, E. P. Gilson, L. Grisham, R. C. Davidson, L. B. Grant Logan, P. A. Seidl, and W. Waldron, "Long plasma source for heavy ion beam charge neutralization," Nucl. Instr. Meth. Phys. Res. A, vol. 606, pp. 124-127, 2009.

[10] R. A. MacGill, M. R. Dickinson, A. Anders, O. R. Monteiro, and I. G. Brown, "Streaming metal plasma generation by vacuum arc plasma guns," Rev. Sci. Instrum., vol. 69, pp. 801-803, 1998. 
[11] P. K. Roy, S. S. Yu, E. Henestroza, A. Anders, F. M. Bieniosek, J. Coleman, S. Eylon, W. G. Greenway, M. Leitner, B. G. Logan, W. L. Waldron, D. R. Welch, C. Thoma, A. B. Sefkow, E. P. Gilson, P. C. Efthimion, and R. C. Davidson, "Drift compression of an intense neutralized ion beam," Phys. Rev. Lett., vol. 95, pp. 234801-4, 2005.

[12] R. L. Boxman, D. M. Sanders, and P. J. Martin, Eds., Handbook of Vacuum Arc Science and Technology. Park Ridge, N.J.: Noyes Publications, 1995, p.^pp. Pages.

[13] A. Anders, Cathodic Arcs: From Fractal Spots to Energetic Condensation. New York: Springer, 2008.

[14] A. I. Morozov and S. V. Lebedev, "Plasma Optics," in Reviews of Plasma Physics, Vol. 8, M. A. Leontovich, Ed., ed New York: Consultants Bureau, 1980, pp. 301-460.

[15] F. F. Chen, Plasma Physics and Controlled Fusion. New York: Plenum Press, 1984.

[16] P. K. Roy, P. A. Seidl, A. Anders, F. M. Bieniosek, J. E. Coleman, E. P. Gilson, W. Greenway, D. P. Grote, J. Y. Jung, M. Leitner, S. M. Lidia, B. G. Logan, A. B. Sefkow, W. L. Waldron, and D. R. Welch, "A space-charge-neutralizing plasma for beam drift compression," Nucl. Instrum. Meth. Phys. Res. A, vol. 606, pp. 22-30, 2009.

[17] A. Anders, I. G. Brown, R. A. MacGill, and M. R. Dickinson, "'Triggerless" triggering of vacuum arcs," J. Phys. D: Appl. Phys., vol. 31, pp. 584-587, 1998.

[18] A. Anders, S. Anders, B. Jüttner, W. Bötticher, H. Lück, and G. Schröder, "Pulsed dye laser diagnostics of vacuum arc cathode spots," IEEE Trans. Plasma Sci., vol. 20, pp. 466-472, 1992.

[19] A. Batrakov, S. Popov, N. Vogel, B. Jüttner, and D. I. Proskurovsky, "Plasma parameters of an arc cathode spot at the low-current vacuum discharge," IEEE Trans. Plasma Sci., vol. 31, pp. 817-826, 2003.

[20] A. Anders and G. Y. Yushkov, "Ion flux from vacuum arc cathode spots in the absence and presence of magnetic fields," J. Appl. Phys., vol. 91, pp. 4824-4832, 2002.

[21] H. R. Griem, Plasma Spectroscopy. New York: McGraw-Hill, 1964.

[22] I. H. Hutchinson, Principles of Plasma Diagnostics Cambridge, UK: Cambridge University Press, 2002.

[23] H. R. Griem, Spectral Line Broadening by Plasmas New York: Academic Press, 1974.

[24] A. Scheeline, G. J. Kamla, and M. J. Zoellner, "Electron concentration measurement in the high voltage spark using Stark broadening of $\mathrm{H}_{\beta}$ emission," Spectrochimica Acta $B$, vol. 39, pp. 677-691, 1984. 


\section{Figure Captions}

Fig. 1 Magnetic field lines of the final focusing solenoid, with the ion beam coming from the left and one of the plasma sources indicated on the right. The density of lines is a measure for magnetic field strength. Since the lines are too dense inside the solenoid, only every $10^{\text {th }}$ line is shown for the off-axis region.

Fig. 2 Set of four plasma sources, each tilted $28^{\circ}$ to the axis as to inject plasma along magnetic field lines.

Fig. 3 On-axis view through a vacuum window, against the beam direction, with the four vacuum arc plasma sources delivering metal plasma.

Fig. 4 Similar view as in fig. 3 showing the coating resulting from the plasma condensation. The plasma was pure aluminum but the thin aluminum film made by a pulse oxidizes to aluminum oxide before the next pulse appears. The colors, visible in the on-line version, are interference colors typical for a thin transparent film deposited on a reflecting substrate.

Fig. 5 Basic experimental arrangement (not to scale): for simplicity, only one of the probe wires is indicated. The photos shown later in this work were taken with the 37-probe array removed and the ring electrode inserted from the left in order to have better visibility to the cathode spots.

Fig. 6 Cross section of the ring-cathode cathodic arc plasma source placed inside the final focusing solenoid (not shown here but in Figs. 1 and 5).

Fig. 7 Magnetic field coil current, arc current and arc voltage as a function of time.

Fig. 8 Top: Overall view on the array of 37 probes mounted on an axially movable shaft; bottom: close-up of the probe's collecting areas.

Fig. 9 Probe circuit for collection of plasma density data; each probe had its own circuit.

Fig. 10 Setup of the single probe.

Fig. 11 Copper plasma produced with an arc current of $270 \mathrm{~A}$; left: in the absence of a magnetic field, and right: at a field strength of 3 Tesla.

Fig. 12 As figure 11 but at the much higher arc current of $4500 \mathrm{~A}$.

Fig. 13 Image of the copper plasma distribution at an intermediate arc current of 500, B-field 3 $\mathrm{T}$; is shows clearly the curved path of the plasma from the cathode spots to the vacuum chamber wall, which is the anode of the discharge.

Fig. 14 Example of density obtained by the 37-probe array: electron density distribution in the $(\mathrm{x}, \mathrm{y})$ plane at $\mathrm{z}=3.5 \mathrm{~cm}$ at time $t=118 \mu \mathrm{s}$ after arc discharge started; the white color indicates a density of $10^{14} \mathrm{~cm}^{-3}$ or greater; arc current $4.5 \mathrm{kA}$, magnetic induction $3 \mathrm{~T}$.

Fig. 15 Example of single probe data, showing the plasma evolution as measured at different 
axial positions, for field of $3 \mathrm{~T}$.

Fig. 16 Emission spectrum of aluminum plasma as measured by the PCM 430 spectrometer when the coil produced a magnetic induction of $3 \mathrm{~T}$. Time integration over one arc pulse.

Fig. 17 High resolution emission spectrum including the $\mathrm{H}_{\beta}$ line for pulsed aluminum plasma in the solenoid with $3 \mathrm{~T}$; time integrated over five arc pulses. 


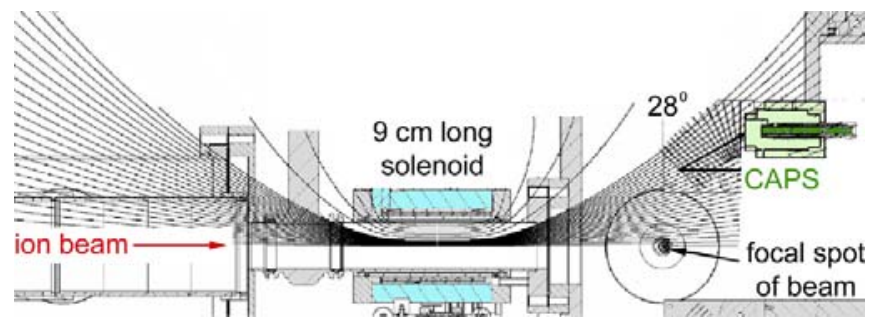

Fig. 1 


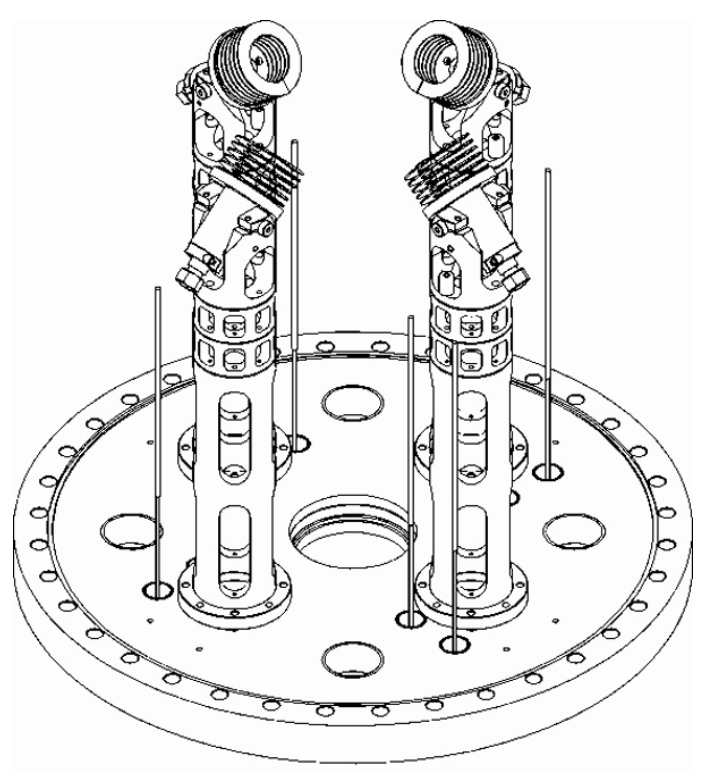

Fig. 2 


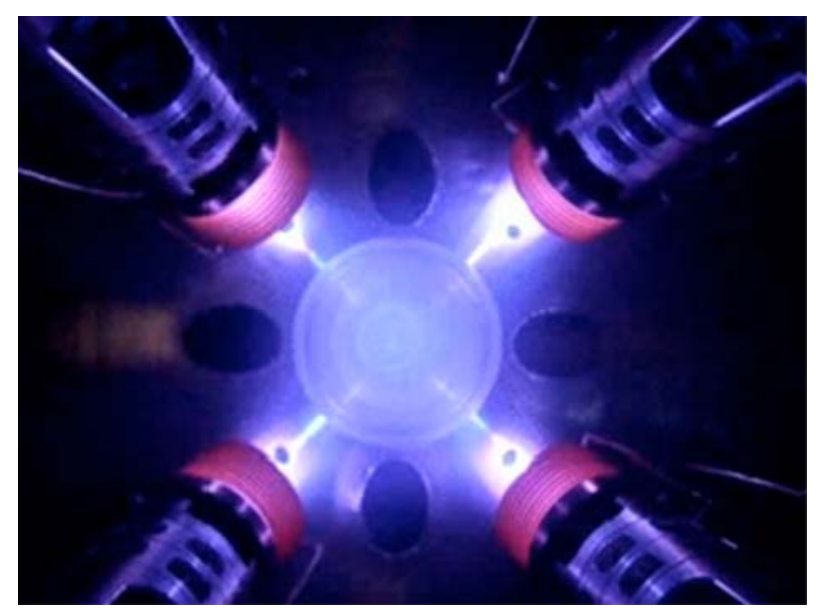

Fig. 3 


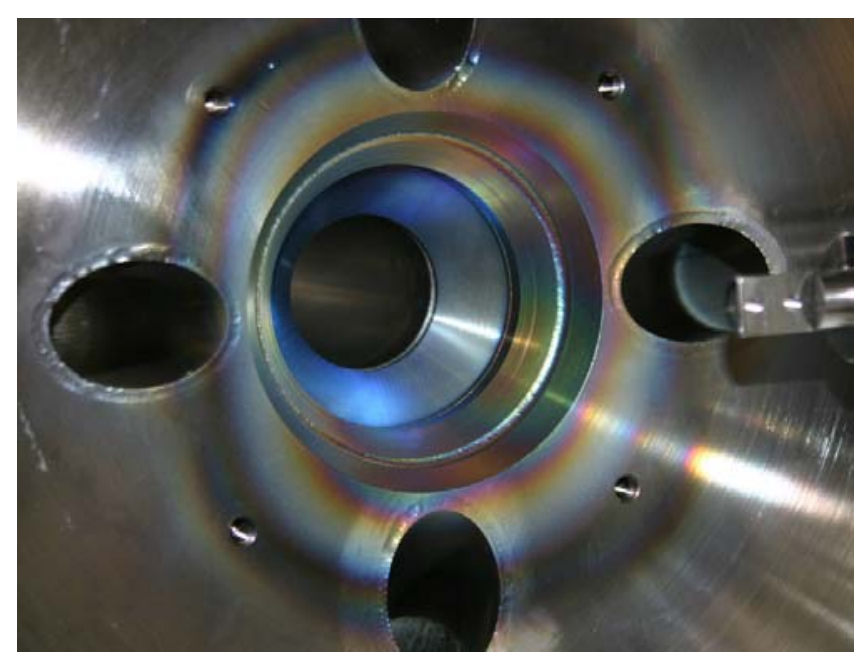

Fig. 4 


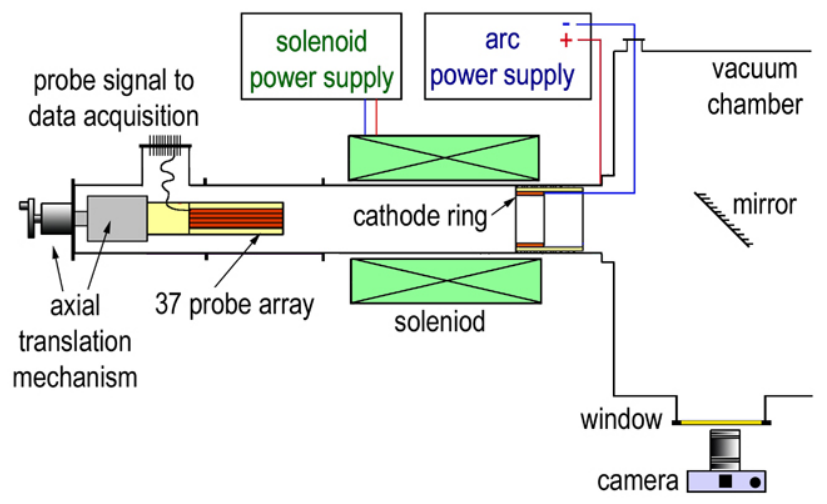

Fig. 5 


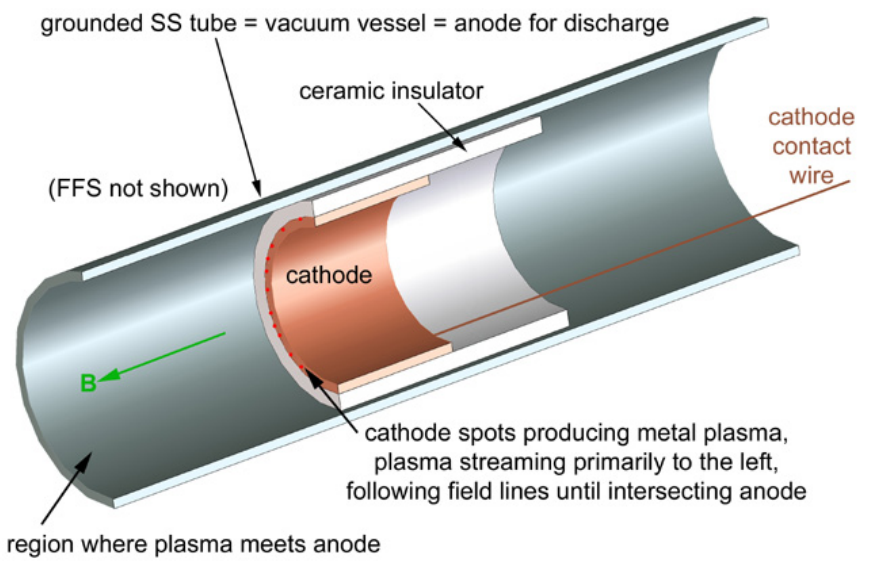

Fig. 6 


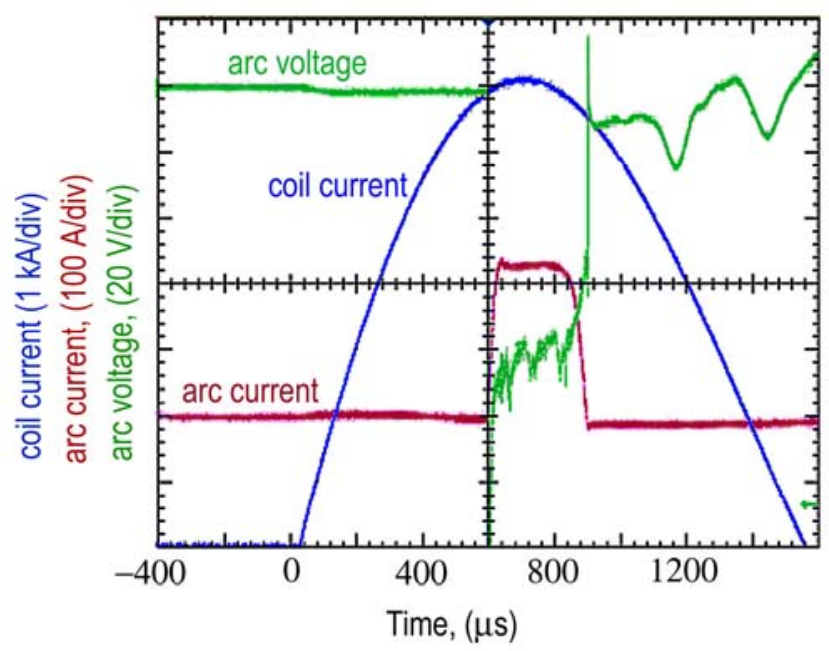

Fig. 7 


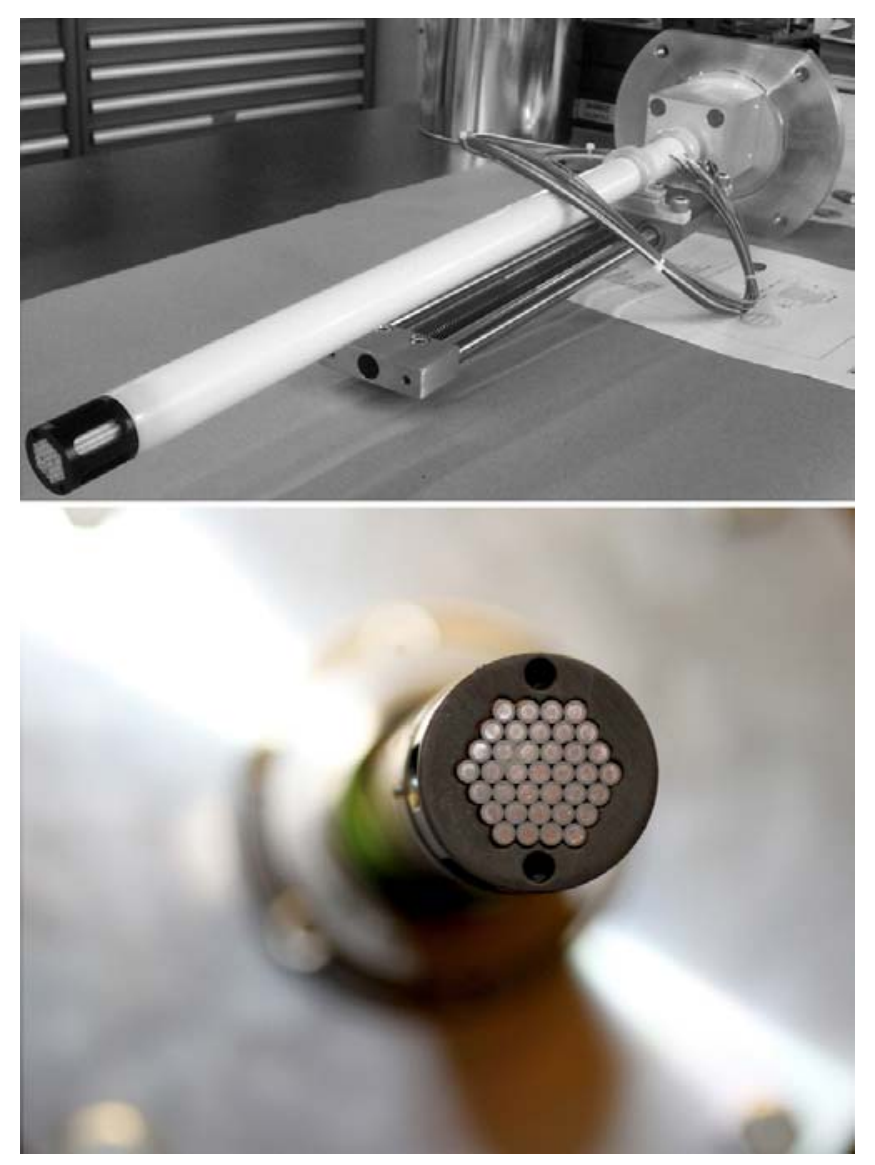

Fig. 8 


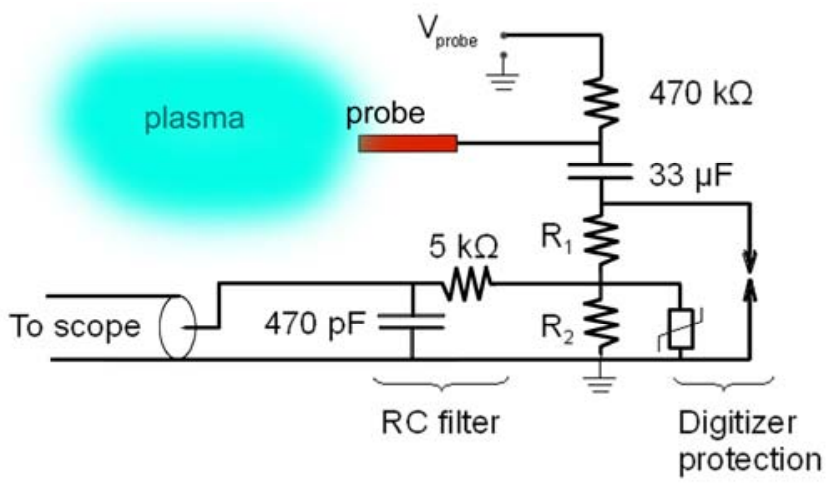

Fig. 9 


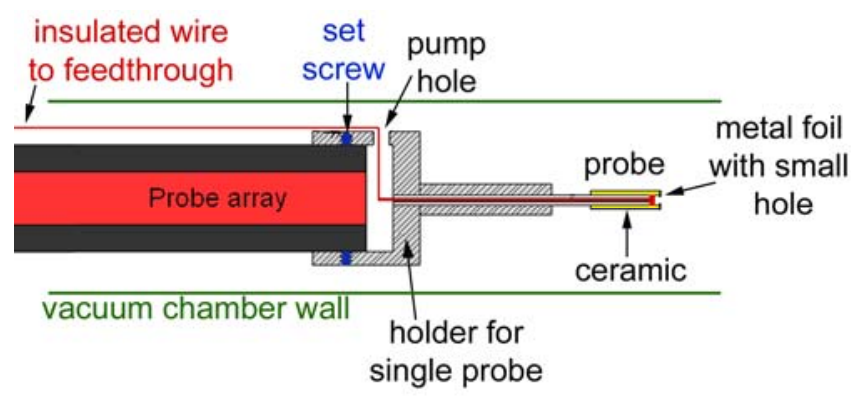

Fig. 10 


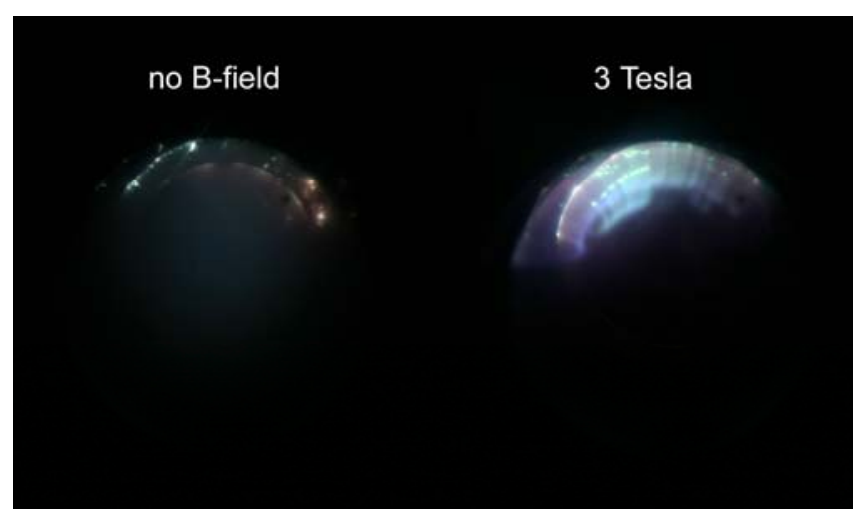

Fig. 11 


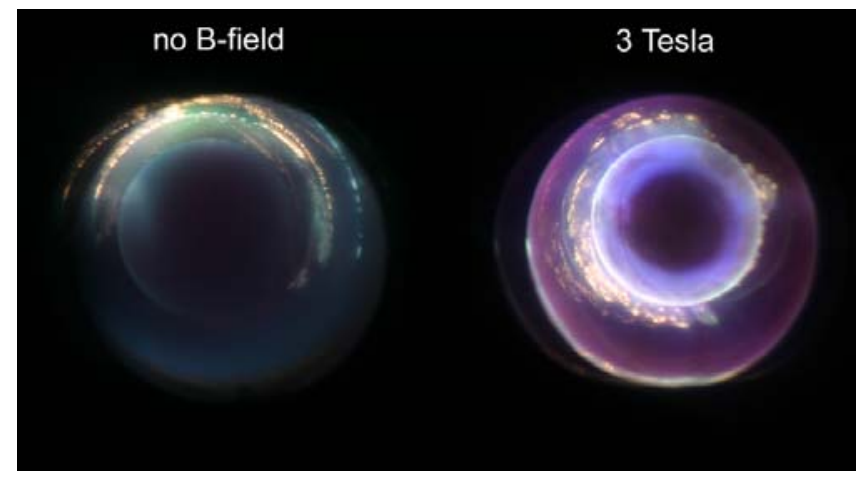

Fig. 12 


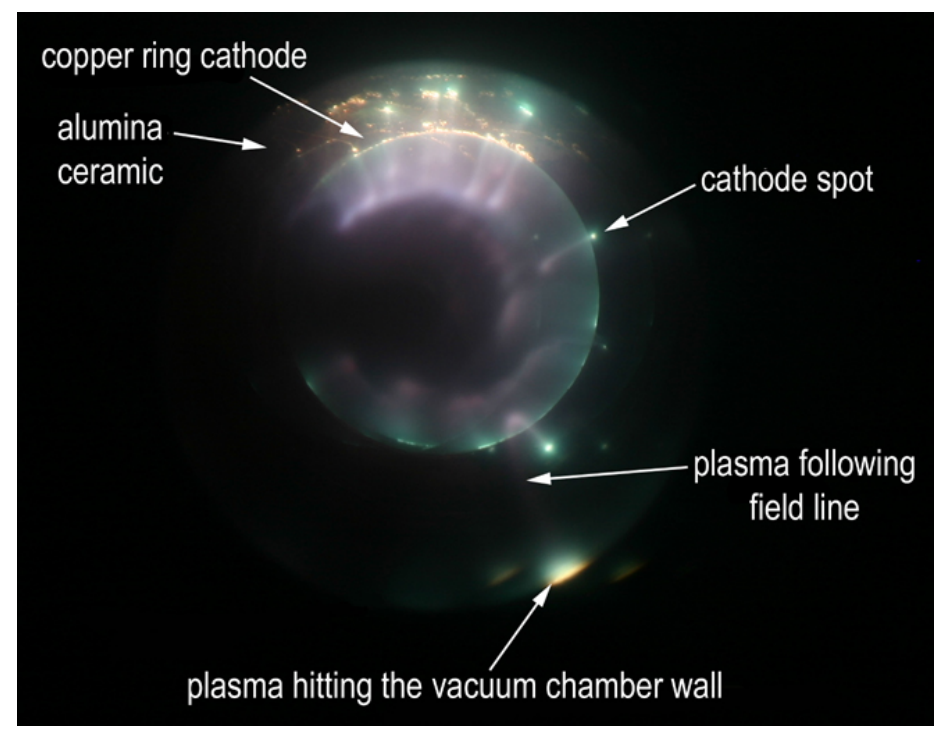

Fig. 13 


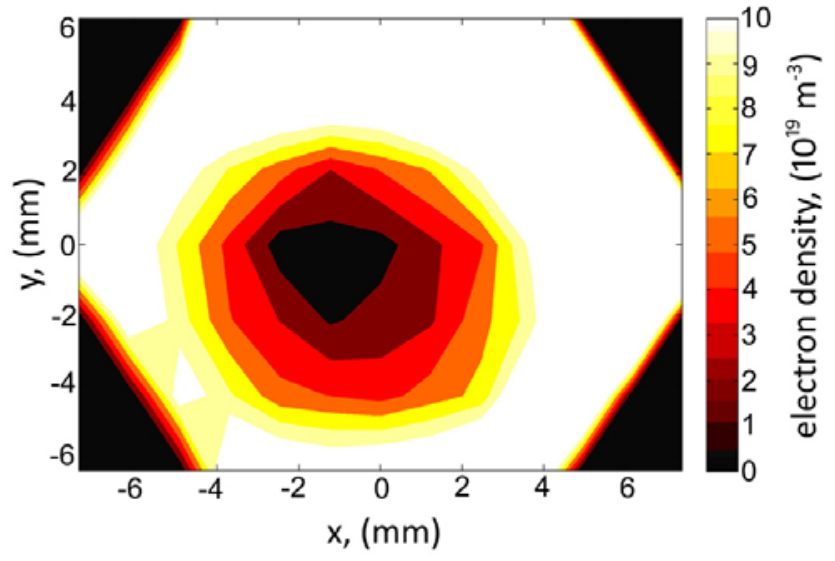

Fig. 14 


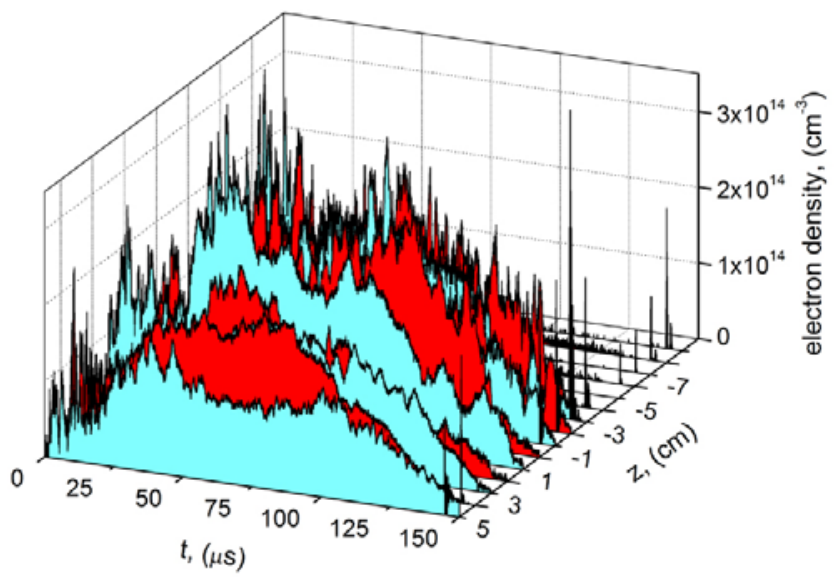

Fig. 15 


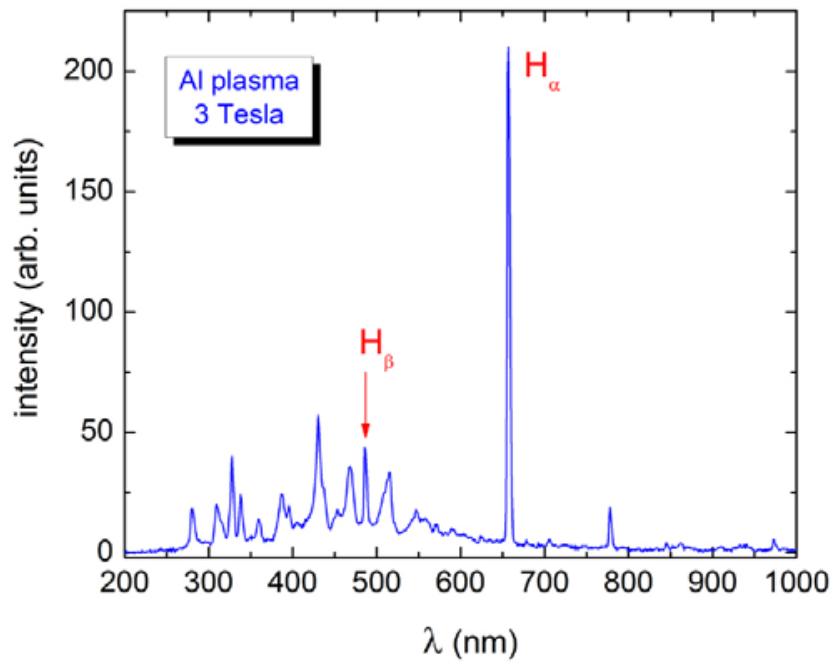

Fig. 16 


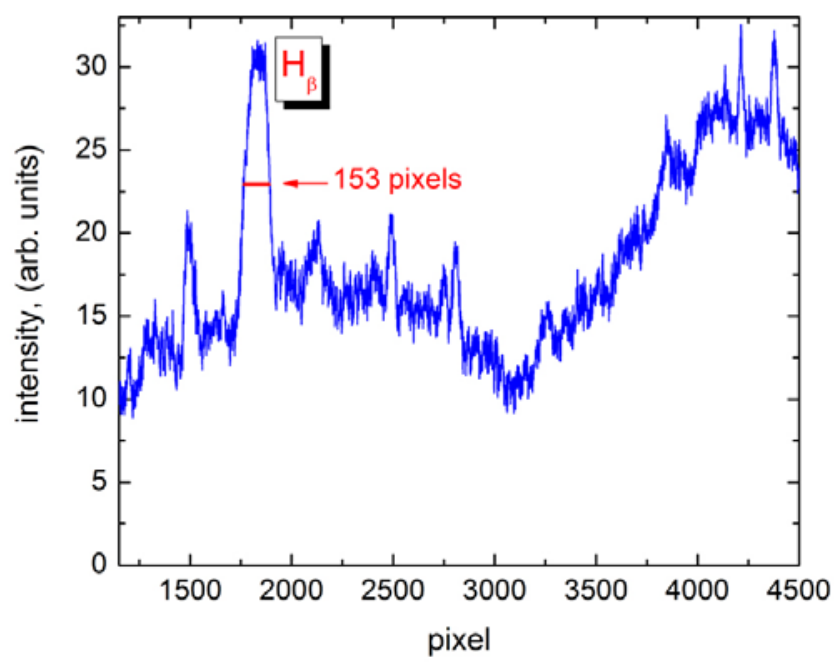

Fig. 17 


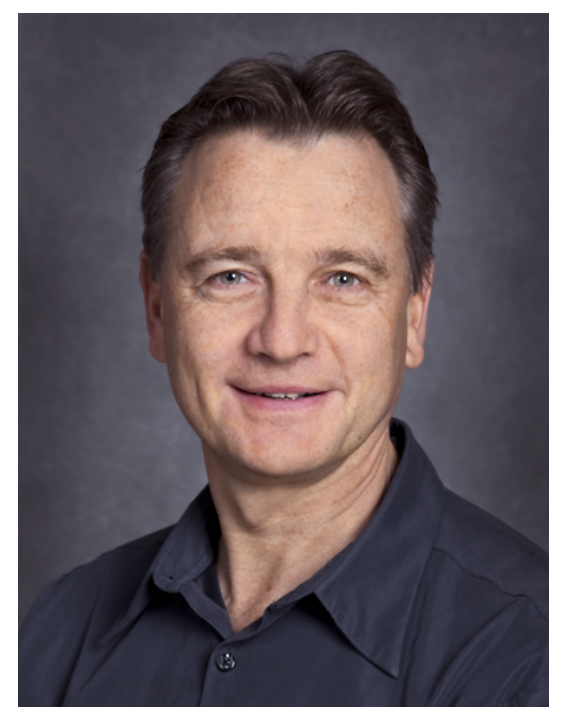

André Anders is a Senior Scientist and the Leader of the Plasma Applications Group at Lawrence Berkeley National Laboratory, Berkeley, California. He studied physics in Germany, Poland, and Russia (then Soviet Union), and received his PhD in 1987 from Humboldt University in East Berlin. From 1987 to 1991 he worked at the Academy of Sciences in East Berlin dealing with discharges in vacuum and electrode-surface phenomena. In 1992 he moved to California to work at Berkeley Lab on various plasma and materials / thin film issues such as the deposition of ultrathin diamond- like carbon films using pulsed filtered cathodic arc plasma technology. He authored three books, including Cathodic Arcs (Springer, NY, 2008), and more than 250 papers in peer-reviewed journals. He serves as Associate Editor for the Journal of Applied Physics, is a member of the Editorial Boards of Applied Physics Letters, the Journal of Applied Physics, and Surface and Coatings Technology, and is chair or member of several conference committees. He received the 2010 IEEE Merit Award of the Nuclear and Plasma Sciences Societies, won two R\&D 100 Awards (1997 and 2009) and was elected Fellow of the American Physical Society (APS), the Institute of Physics (IoP of UK), and the Institute of Electrical and Electronics Engineers (IEEE). 


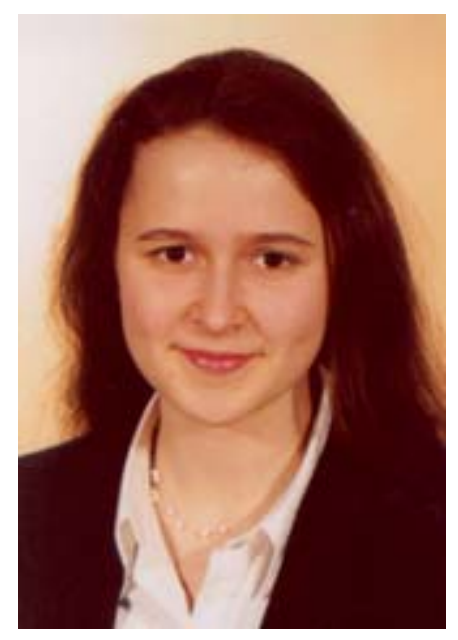

Marina Kauffeldt is currently a PhD student at the Lab for Plasma Technology at the University of Federal Armed Forces in Munich, Germany. She is the recipient of a civilian fellowship of the Siemens company. She studied mathematical engineering, a combined study of mathematics and electrical engineering, at the University of Federal Armed Forces, where she received her diploma degree in January 2010. As part of studies she spent a trimester at the Lawrence Berkeley Laboratory working on a project at the Plasma Application Group working in the field of thin film deposition using filtered cathodic arc plasma technology. 


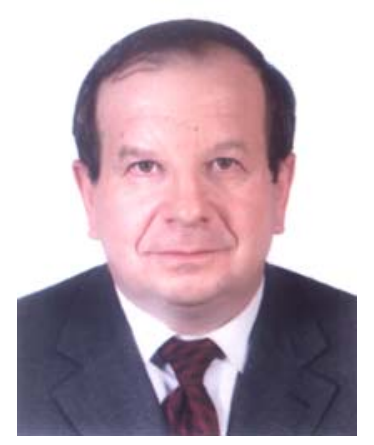

Efim M. Oks received his PhD degree in physical electronics in 1985 and the D.Sc. degree in vacuum and plasma engineering in 1994, both from the High Current Electronics Institute, Russian Academy of Sciences, Tomsk, Russia. At the current time he is a Senior Physicist and the Leader of the Plasma Sources Department at the High Current Electronics Institute and Professor and Head of the Physics Department at Tomsk State University of Control Systems and Radioelectronics. He is the author or coauthor of about 140 papers in the area of basic phenomena in low pressure gaseous discharges and vacuum arc plasmas, charged particle emission from low temperature plasma, ion sources and plasma electron guns, and a range of plasma applications. 


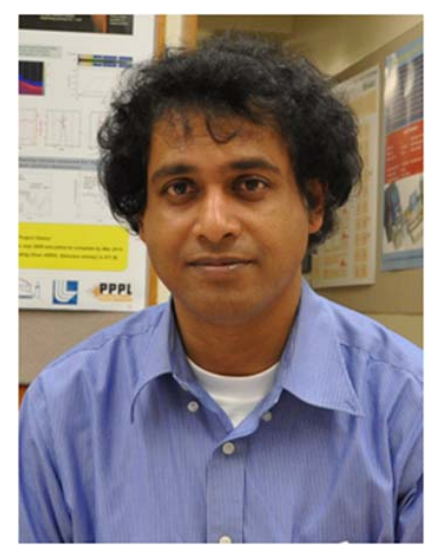

Prabir K. Roy has been working with the Accelerator and Fusion Research Division at Lawrence Berkeley National Laboratory (LBNL), California, USA, since 2002. He was engaged in two post-doctoral physicist positions: one with Louisiana Tech University for Fermilab's D-zero project, and the other with the University of California for LBNL's NTX project. Prabir received his Ph. D. degree from ILE, Osaka University, Japan (1998) in Electromagnetic Energy Engineering Physics. He received Bachelor of Science (Honors) and Master of Science degrees, both in Physics, from Chittagong University, Bangladesh. His experience in experimental physics relates to charged particle beams, plasmas, lasers, and electromagnetism. Prabir is a current member of APS, IEEE, and AAAS. Over the course of his career he has published numerous scientific papers in internationally-circulated, peer-reviewed journals, presented at international conferences; acted as a referee for prominent journals in the field; and taught Physics at the University of California Berkeley Extension. 